\title{
Update on Islet Transplantation
}

\author{
Michael McCall and A.M. James Shapiro \\ Clinical Islet Transplant Program and Department of Surgery, University of Alberta, Edmonton, \\ Alberta T6G 2B7, Canada \\ Correspondence: amjs@islet.ca
}

Clinical islet transplantation has progressed considerably over the past 12 years, and $>750$ patients with type 1 diabetes have received islet transplants internationally over this time. Many countries are beginning to accept the transition from research to accepted and funded clinical care, especially for patients with brittle control that cannot be stabilized by more conventional means. Major challenges remain, including the need for more than one donor, and the requirement for potent, chronic immunosuppression. Combining immunological tolerance both to allo- and autoantigens, and a limitless expandable source of stem cell- or xenograft-derived insulin-secreting cells represent remaining hurdles in moving this effective treatment to a potential cure for all those with type 1 or 2 diabetes.

$T_{\mathrm{p}}^{\mathrm{h}}$ he transplantation of islets of Langerhans, procured from a deceased donor, has become a promising treatment for type 1 diabetes mellitus (T1DM). Currently, C-peptide negative patients are considered for this procedure if they display severe glycemic lability, recurrent hypoglycemic episodes, and a reduced ability to detect the symptoms of hypoglycemia. It is estimated that patients with T1DM experience 1.3 severe hypoglycemic episodes per year, with 5\% of patients accounting for $54 \%$ of these episodes (Pedersen-Bjergaard et al. 2004). It is these patients that benefit most from islet transplantation and in whom chronic immunosuppression can be justified. The indications are expanding, with microvascular and even macrovascular complications improving after transplantation (Warnock et al. 2008; Thompson et al. 2011).

In brief, islet transplantation involves the procurement of a donor pancreas with subse- quent digestion and separation of the exocrine tissue and stroma from the islets. This is performed using enzyme degradation and density centrifugation. Isolated islets are assessed for quantity and quality (Fig. 1). Islet preparations that contain an adequate number of islets (5000 islet equivalents [IEQ] per kilogram based on recipient weight) are then transplanted into a diabetic recipient. The majority of transplants involve percutaneous cannulation of a branch of the portal vein with subsequent gravity infusion of the islet preparation (Fig. 2). Recipients are placed on immunosuppression and other medications designed to enhance islet engraftment and survival.

The first attempt at islet transplantation occurred in 1893 when Watson-Williams and Harsant transplanted minced sheep's pancreas into the subcutaneous tissue of a young boy with ketoacidosis (Williams 1894). The discovery of

Editors: Jeffrey A. Bluestone, Mark A. Atkinson, and Peter R. Arvan

Additional Perspectives on Type 1 Diabetes available at www.perspectivesinmedicine.org

Copyright (C) 2012 Cold Spring Harbor Laboratory Press; all rights reserved; doi: 10.1101/cshperspect.a007823

Cite this article as Cold Spring Harb Perspect Med 2012;2:a007823 


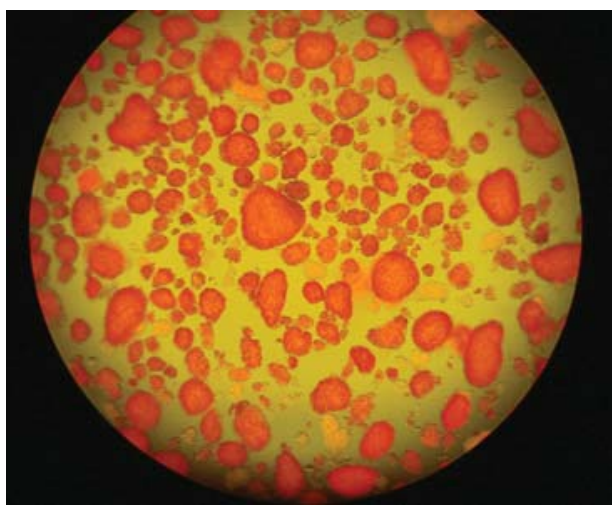

Figure 1. Isolated and purified human islets of Langerhans, postculture, ready for transplantation.

insulin reduced interest in transplantation as a treatment for diabetes, but was renewed in 1972 when Drs. Ballinger and Lacy demonstrated a method for isolating intact islets of Langerhans from rodents with subsequent in vivo function after transplantation in a rodent diabetes model (Ballinger and Lacy 1972). Nearly 20 years later, Scharp et al. (1990) achieved the first case of clinical insulin independence, which lasted nearly 1 month. This achievement was possible owing to advances in islet isolation and purification including the development of the Ricordi chamber by Dr. Camillo Ricordi in 1989 (Ricordi et al. 1989). Further advances and experimentation culminated in the publication of the Edmonton Protocol in 2000 (Shapiro et al. 2000). In this series of seven patients with T1DM receiving islets from multiple (2-4) donors, $100 \%$ of recipients achieved insulin independence. This study heightened the interest in islet transplantation and proposed the use of a steroid-free immunosuppression protocol, the transplantation of a larger islet mass $(>11,000$ IEQ $/ \mathrm{kg}$ recipient weight) and the avoidance of islet culture before transplantation. Although islets are now routinely cultured, steroids continue to be avoided in posttransplant immunosuppression regimens. The use of multiple donors, while enabling the transplantation of a large islet mass, limits the number of patients that can receive grafts. Current data on the outcomes of islet transplantation show insulin-independence rates to be $>27 \%$ at $2 \mathrm{yr}$ posttransplant (Inves- tigators 2009). The Edmonton group and others have observed an inexorable decline in insulin independence out to $8 \mathrm{yr}$ posttransplant, but with persistent C-peptide secretion and complete protection from hypoglycemic reactions in $>70 \%$ of recipients (Fig. 3). This is through the use of multiple islet donors. Even more recent data hints at 3-yr insulin-independence rates nearing $50 \%$.

A major international trial was initiated by the Immune Tolerance Network (ITN) both to replicate Edmonton's success, and to set up a series of clinical sites for future trials in tolerance and islet transplantation. The major findings of that trial were that insulin independence was indeed attainable at multiple sites where a common islet isolation and clinical immunosuppressive protocol was applied, but variance in success between sites reflected site-specific experience both with the complex preparation of human islets for transplantation, and clinical experience in the routine management of immunosuppressive therapies, especially around dosing of sirolimus (SRL) (Shapiro et al. 2003, 2006). Enabling lower islet masses from single donors to achieve insulin independence is one of the current goals in islet transplantation and would allow transplantation of a wider group of recipients and advance islet transplantation as the "treatment for all" in diabetes. In addition, the achievement of longer-term insulin independence is highly sought. Although independence

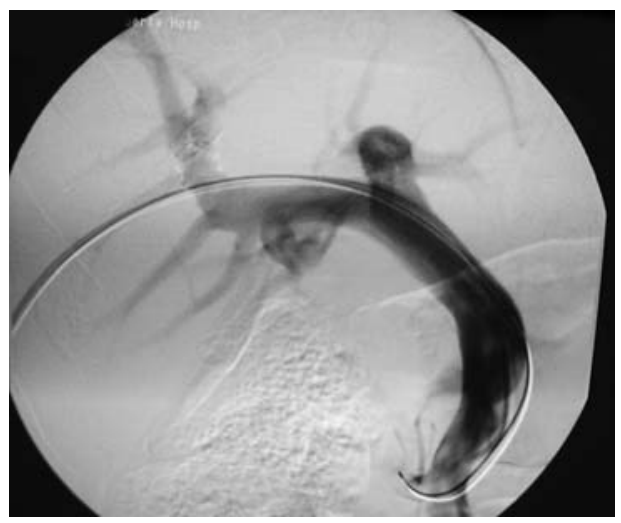

Figure 2. Portal angiogram after percutaneous transhepatic portal access, with catheter tip in the superior mesenteric vein, ready for islet infusion. 


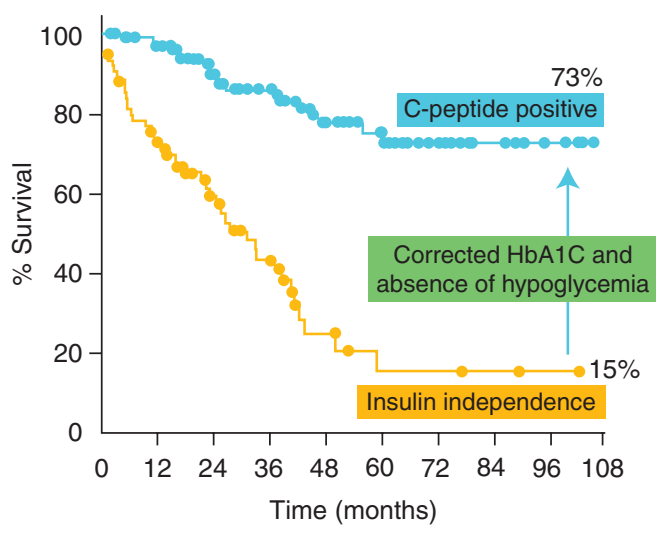

Figure 3. Nine-year insulin independence and C-peptide islet graft function rates with the original Edmonton Protocol immunosuppression. (Data from the University of Alberta.)

out to $10 \mathrm{yr}$ is possible (Berney et al. 2009) this is certainly not standard. The focus of this work is to review the advances in islet transplantation that have made the current results possible and to look ahead at some of the future advances aimed at achieving these goals.

\section{SINGLE-DONOR ISLET TRANSPLANTATION}

Although the majority of islet transplant recipients receive islets from multiple donors (average 2-3), this limits the number of transplants that can occur. The success of the Edmonton Protocol can be attributed, in part, to the transplantation of a large islet mass ( $>11,000$ IEQ/ $\mathrm{kg}$ ) (Shapiro et al. 2000). There are numerous factors in the pre- and posttransplant period that contribute to islet loss. Up to $60 \%$ of the islet mass can be lost in the first few days following islet infusion (Biarnes et al. 2002). The use of a larger islet mass over multiple islet infusions leads to a larger mass of surviving $\beta$ cells and a higher likelihood of insulin independence. However, donor limitations reduce the number of patients that can be treated.

Achieving posttransplant islet function and even insulin independence with single donors would help to transition islet transplantation from therapy for few to a cure for all with T1DM. It would also avoid exposing the recip-
Update on Islet Transplantation

ient to polyhuman leukocyte-associated (HLA) antigens that may preclude future transplants for the recipient. Single-donor islet transplant success has been achieved in certain centers, most notably at the University of Minnesota (Hering et al. 2004, 2005) and University of California, San Francisco (UCSF) (Posselt et al. 2010a,b). However, this is not a universal phenomenon. The University of Alberta, one of the most active islet transplant centers worldwide with $>300$ intraportal islet infusions in $>150$ subjects over the past 12 years, despite making progress in long-term outcomes does not routinely achieve single-donor islet engraftmentin fact, this is a rare occurrence in only $10 \%-$ $15 \%$ of cases in Edmonton's hands. Exactly which factors account for center differences in single-donor islet transplant success is difficult to define with precision, especially in the absence of common predictive potency assays for comparison. Important differences between Edmonton's single-donor experience and that of selected centers in the United States likely reflect two dominant factors: (1) Edmonton is relatively nonselective in which pancreas organs are processed for islet transplantation, the philosophy being to maximize utility of any organ not used for whole pancreas transplantation in Canada; and (2) real differences in organ donor parameters between Canada and the United States-in Canada it is exceedingly rare to have access to young donors with high body mass index (BMI), donors which are more favored in the United States islet transplant centers as they yield superior numbers of islets of increased potency. Differences in the funding mechanism further polarize this, as scarce research dollars must cover the costs of all islet isolations in the United States (pancreas procurement fees of up to $\$ 35,000$ and islet processing fees of up to $\$ 40,000$ ), whereas in Canada there are no procurement fees, and the costs of islet processing are covered by the public healthcare system. Furthermore, the cold ischemic time for transfer of a pancreas from donor explant to processing in the islet center tends to be much longer in Canada than in the United States, because islet processing is centralized in Edmonton and in Vancouver, often with cold 
ischemic times exceeding $12 \mathrm{~h}$, which may contribute substantially to the potency of the final islet product. Beyond these obvious differences, it is difficult to identify other major contributory factors, as the islet processing and culture techniques are common and shared between centers, including specific batches of optimized collagenase blend enzymes. Ongoing analysis of data from the Collaborative Islet Transplant Registry (CITR) is anticipated to highlight these differences with more precision over time.

\section{ADVANCES IN ISLET PROCUREMENT AND ISOLATION}

\section{Pancreas Donor Access and Selection}

The selection of a donor pancreas for islet isolation is a critical step in the transplantation procedure. Success is dictated by a number of factors including donor BMI, cold ischemia time, use of vasopressors, and blood glucose levels (Zeng et al. 1994; Matsumoto et al. 2004; Nano et al. 2005). Attempts to standardize the donor selection process have been undertaken using algorithmic scoring systems (O'Gorman et al. 2005; Witkowski et al. 2006). However, these have been correlated with islet isolation success and not necessarily posttransplant islet function. The selection of an "ideal" donor, leading to a large postisolation islet mass would increase the success of single-donor transplantation. However, the decision to allocate the "optimal" pancreas to islet isolation must be made against the decision to use it for whole-organ pancreas transplantation, which should still take priority in most centers, because this is a more established procedure with more durable single-donor success, albeit with a much greater burden of perioperative risk. As the gap closes in insulin-independent success between pancreas alone and islet transplantation alone (ITA) (and that gap has almost closed with recent data from Minnesota, Edmonton, Geneva, Lille, and at UCSF showing $50 \%$ insulin independence at $5 \mathrm{yr}$ ), it is likely that pancreas allocation rules will change further to allow more optimal pancreas organs to be allocated to islet processing.
Most islet isolations begin with a pancreas obtained from a heart-beating brain dead organ donor. Most donors fall into the "marginal" category, which negatively affects islet yield and potency. The use of donors that have succumbed to cardiac death, more generally accepted in the Netherlands, have yielded successful outcomes in some centers, most notably the University of Pittsburgh (Markmann et al. 2003; Saidi et al. 2010). Living donors are used in many transplant programs including those performing liver and kidney graft transplants but have failed to gain popularity in islet transplantation, or indeed in segmental vascularized pancreas transplantation although technically possible (as shown by Sutherland and the Minnesota group). The group in Kyoto successfully transplanted islets from mother to daughter using a distal pancreatectomy in the donor (Matsumoto et al. 2005). This places the donor at risk for complications including pancreatic leak, fistula, and diabetes, making this an unpopular approach for many. The potential need for multiple donors creates an added detraction in the setting of living islet donation. Limited experience in this area has yet to establish whether procurement under ideal circumstances (absence of brain death and inotropic support) and use of unpurified but limited volume pancreatic digest from half a pancreas (similar to an islet autograft, which does have increased rates of single-donor engraftment), will increase rates of single, living donor insulin-independent success.

\section{Islet Isolation}

Islet isolation involves two distinct steps: pancreas digestion and islet purification. Pancreas digestion begins with sectioning of the pancreas, then exposing it to enzymatic digestion within a Ricordi chamber, developed nearly 20 years ago by Ricordi et al. (1989). Even though the apparatus itself has not changed, the enzyme cocktails have. Early enzymes varied widely and provided inconsistency owing to lot-to-lot variability (Kin et al. 2007). This was temporarily resolved with the development of the enzyme blend Liberase HI by Roche (Roche Applied 
Update on Islet Transplantation

Science, Indianapolis, IN). Unfortunately, reports surfaced detailing the use of bovine brain components in its manufacturing, leading programs to remove it from their protocols. Recent state-of-the art blends include collagenase NB1 (Serva, Heidelberg, Germany), a mammalian tissue-free version of Liberase (Liberase MTF, Roche Applied Science, Indianapolis, IN) and a new blend that enhances the proportion of intact $\mathrm{C} 1$ collagenase (VitaCyte CIzyme collagenase HA enzyme) (Szot et al. 2009; Brandhorst et al. 2010; O'Gorman et al. 2010). At the current time, the Serva, Roche, and VitaCyte products perform with similar success, but head-to-head comparisons are pending to more optimally define merit.

\section{Islet Purification}

Density gradient islet purification reduces the volume of tissue infused into the portal vein. Infusions of large volumes have been shown previously to lead to thrombosis, embolism, and very rarely death (Mehigan et al. 1980; Mittal et al. 1981). It should be noted that in the post-Edmonton era, there have been no reports of acute death resulting from intraportal islet infusion and disseminated intravascular coagulation, which is a testament to islet purification, current systemic anticoagulation protocols, and especially to the use of low-endotoxin-containing enzyme products. Islets are purified based on their differential density compared to the surrounding pancreatic exocrine tissue. The development of the COBE 2991 (COBE Laboratories, Inc., Lakewood, CO), originally designed for blood separation, has made this separation more reproducible, shortened isolation times, and increased islet purity (Lake et al. 1989). Originally, most density gradients were composed of Ficoll, a synthetic polymer of sucrose, or a derivative of it. Newer gradients have improved on this. Kin et al. (2008) were able to achieve a $77 \%$ islet recovery rate using University of Wisconsin solution with Biocoll. Another promising compound is Iodixanol. Alternatively used as a radiologic contrast agent under the name Visipaque, Iodixanol (Optiprep) can enhance islet recovery (Noguchi et al. 2009) and reduce chemokine/cytokine production (Mita et al. 2010). Iodixaniol has seen increasing use recently and may contribute in some (but not all) single-donor islet transplant protocols (Matsumoto et al. 2011).

\section{ADVANCES IN ISLET IMPLANTATION}

The vast majority of islet transplants have been performed percutaneously into the portal vein system (Fig. 2). Ideally, islets require ready access to a robust vascular supply including oxygen and glucose and the means to deliver insulin to target tissues. From the patient's standpoint the transplantation of islets should confer minimal procedural risk and allow for long-term function. In addition, having a means to monitor islets posttransplant could allow interventions to promote graft recovery and engraftment.

\section{The Portal Site}

Although implantation of islets directly into the portal bloodstream with their eventual settlement in the smaller venules yields a high oxygen tension and a near-physiologic insulin delivery environment, it also exposes islets to very detrimental blood components. The instant bloodmediated inflammatory reaction (IBMIR), characterized by rapid activation of the coagulation and complement systems leads to rapid islet loss (Bennet et al. 1999, 2000). Anticoagulation with heparin posttransplant has helped to reduce the impact of IBMIR and improved the success rate of single-donor islet engraftment from $10 \%$ to $40 \%$ (Koh et al. 2010). Further strategies to overcome this barrier to successful islet engraftment are continuously sought, including blockade of tissue factor (Johansson et al. 2005) and further anticoagulant strategies.

This procedure is not without complications. The Edmonton group reported complications including bleeding (8\%), portal vein thrombosis (3\%), and bile leakage (Owen et al. 2003). The risk of bleeding has been substantially reduced through the use of agents that plug the catheter tract (including Avitene paste and coils) (Villiger et al. 2005). Inclusion of heparin in the islet preparation and intravenously 
M. McCall and A.M. James Shapiro

postinfusion has made life-threatening thrombosis extremely rare.

Posttransplant monitoring of intraportal islets, although potentially useful for guiding therapy, has proven difficult. Random biopsy sampling is only successful in $30 \%$ of attempts and may not provide timely answers as samples are prepared and analyzed (Toso et al. 2009). Imaging modalities may provide a more timely answer to posttransplant islet status. Magnetic resonance imaging (MRI), using islets labeled with a paramagnetic compound, can provide relatively high spatial resolution but lacks long-term application and may be limited by iron overload (Toso et al. 2008; Medarova and Moore 2009). Positron emission tomography (PET) using $\left[{ }^{18} \mathrm{~F}\right] \mathrm{FDG}$-labeled islets may provide superior sensitivity and specificity as compared to MRI and has already elucidated the early posttransplant loss of islets (Eich et al. 2007a; Eriksson et al. 2009). However, longer- term monitoring of islets in the portal site has remained elusive owing to islet dispersion and washout of tracers.

Although the portal site remains the most common islet habitat, it is certainly not without its drawbacks. Early islet loss and difficulties in posttransplant monitoring have led research groups to search for alternatives.

\section{ALTERNATIVE TRANSPLANT SITES}

The drawbacks of the portal site, namely, the early posttransplant islet loss and the difficulty in reliably monitoring the graft, have initiated a search for alternative sites (Fig. 4). In a mouse model, syngeneic rodent islets transplanted into the femur are able to reverse hyperglycemia without any evidence of derangement of the hematopoietic system or disruption of structural integrity (Cantarelli et al. 2009). Another promising site is the gastric submucosal space

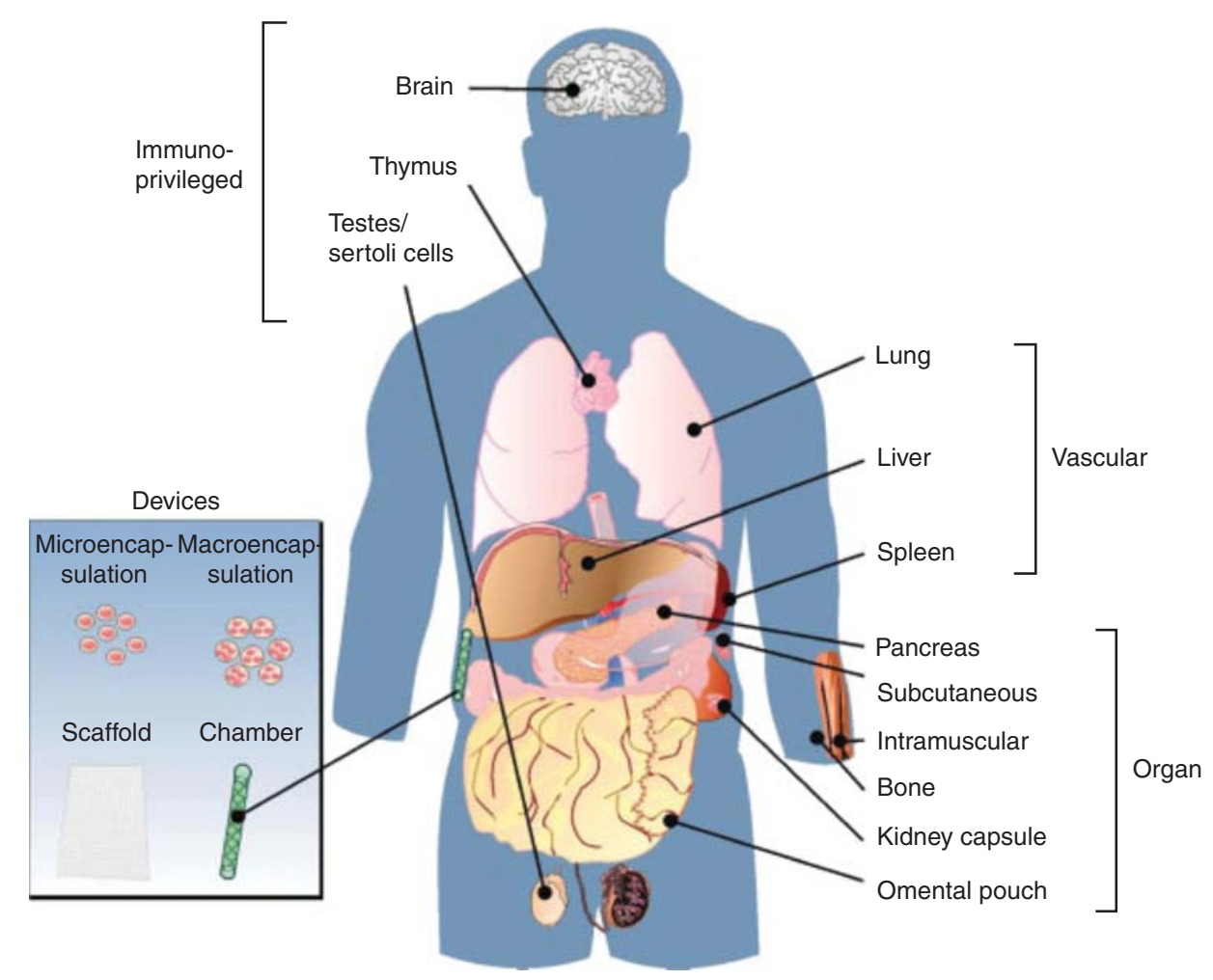

Figure 4. Alternate sites for islet implantation. (Figure from Merani et al. 2008a; reprinted, with permission, from the author.) 
(GSMS) (Echeverri et al. 2009; Wszola et al. 2009). After endoscopic placement, islets would benefit from a rich blood supply and a high oxygen tension. Preclinical trials using a porcine model showed reduced early islet loss and insulin requirements when GSMS islets were compared to intraportal islets (Echeverri et al. 2009).

Transplantation into a more readily accessible site, such as skeletal muscle, would facilitate graft monitoring, implantation, and biopsy. In mice, intramuscular islet grafts display enhanced revascularization with vessel densities and blood flow comparable to the native pancreas (Christoffersson et al. 2010). Clinically, a group in Sweden transplanted a 7-yr-old girl with autologous islets (6400 islets $/ \mathrm{kg}$ ) after total pancreatectomy (Rafael et al. 2008). The techniques developed using PET in intraportal implantation would apply readily to intramuscular grafts provided the correct biomarkers were available (Eriksson and Alavi 2011). One drawback of the muscular site is the space limitation. Combination of the portal and intramuscular site would allow placement of a "sentinel" graft in muscle to allow for early detection and intervention in the case of rejection.

A novel approach to graph implantation is the use of islet encapsulation devices. Both micro- and macroencapsulation devices have been developed that provide a secure oxygen and nutrient-rich environment for islets, while potentially affording a degree of immunoisolation. This idea dates back to 1977 when Chick et al. (1977) developed an "artificial pancreas," a small hollow tube made of semipermeable acrylic copolymer. When filled with islets, these devices restored glycemia for hours before their removal. Others have used alginate and related compounds with improved success, showing robust enough protection to allow islet xenograft survival (Duvivier-Kali et al. 2001, 2004; Omer et al. 2003). Macroencapsulation, providing a larger volume for tissue implantation, has the added advantage of being reloadable and retrievable. One example is the TheraCyte device that is composed of a double membrane of polytetrafluoroethylene (PTFE) (Suzuki et al. 1998; Yang et al. 2002). The inner membrane provides immunoisolation, whereas the outer membrane facilitates angiogenesis. Despite the advances in device conception and construction, there has been a challenge to achieve success in large animal allogeneic or xenogeneic islet models. Scharp et al. have reported protection of islets for 2 weeks in macro devices transplanted into normal, type 1 , and type 2 diabetic patients (Scharp et al. 1994). More recently, Calafiore et al. (2006) have shown function of microencapsulated allogeneic islets transplanted intraperitoneally, whereas Elliott et al. (2007) reported a case of macroencapsulated porcine islets showing live islet cells nearly 10 years after implantation into a diabetic recipient. Although islet encapsulation is a promising avenue, it is still hampered by challenges including overcoming device biocompatibility, ineffective immunoprotection, hypoxia, and posttransplant inflammation (Vaithilingam and Tuch 2011).

\section{ADVANCES IN POSTTRANSPLANT TREATMENT}

Islet implantation into a recipient with diabetes exposes the islets to a number of detrimental factors. These include IBMIR, hypoxia, inflammation, hyperglycemia, mechanical injury, and immune-meditated islet attack. The aim of recipient treatment posttransplant is to reduce the effects these factors have on the transplanted islet mass. On the horizon there are several exciting and innovative trials moving forward in the clinical arena in islet transplantation, and in fact there are $>80$ registered trials presently registered with ClinicalTrials.gov (Table 1). With $>1500$ subjects potentially enrollable in these trials, and at an estimated cost of in excess of $\$ 150,000$ USD per subject treated, the burden of cost on research organizations is enormous (\$227 million USD), and is likely not sustainable. There are major endeavors under way now in the United States to secure a biological license (BLA) for islet transplantation so that many of the routine costs of islet preparation and patient care can be assumed by reimbursable sources (e.g., Medicare and Medicaid). In Canada, this process occurred in 2001, with the Canadian Provincial governments reimbursing islet transplantation as standard clinical care in carefully 
M. McCall and A.M. James Shapiro

Table 1. Current active clinical trials in islet transplantation registered with Clinical Trials.gov ( $>80$ trials)

\begin{tabular}{|c|c|c|c|c|c|c|c|c|c|c|}
\hline Trial ID\# \# & las & stigator & cation & ype & irol & Statu & $\mathrm{Ca}$ & Intervention & & BLA \\
\hline $\begin{array}{l}\text { NCTroo2147866 } \\
\text { NCT00579371 }\end{array}$ & & $\begin{array}{l}\text { Marlon Levy } \\
\text { Brian Stevens }\end{array}$ & $\begin{array}{l}\text { Baylor } \\
\text { Nebraska }\end{array}$ & $\begin{array}{c}\text { ITAMAK } \\
\text { ITA }\end{array}$ & 15 & $\begin{array}{l}\text { Compleated } \\
\text { active }\end{array}$ & $\begin{array}{l}\text { IImmunosupp } \\
\text { Immunosupp }\end{array}$ & Thymo-single donor & & \\
\hline $\begin{array}{l}\text { NoTro006552 } \\
\text { NCT00679042 }\end{array}$ & III & $\begin{array}{l}\text { Ken Bramman } \\
\text { Jose Obernolzer }\end{array}$ & $\begin{array}{c}\text { Virginia } \\
\text { uic }\end{array}$ & ITAAK & $\begin{array}{l}20 \\
50\end{array}$ & $\begin{array}{l}\text { Active } \\
\text { Active }\end{array}$ & $\begin{array}{l}\text { Immunosupp } \\
\text { Enngrattent } \\
\end{array}$ & Exenatide & Yes & Yes \\
\hline 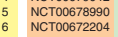 & I & $\begin{array}{l}\text { Gunnar Turesson } \\
\text { Bembnarar Hering }\end{array}$ & 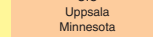 & 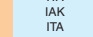 & $\begin{array}{l}10 \\
10\end{array}$ & $\begin{array}{l}\text { Pending } \\
\text { Suspondead }\end{array}$ & $\begin{array}{l}\text { Engrattment } \\
\text { Immonosporession }\end{array}$ & $\begin{array}{l}\text { Heparin coating of istets (IBMIR) } \\
\text { Rapativa (efirizumab and SRL }\end{array}$ & & \\
\hline $\begin{array}{l}\text { NCT002025233 } \\
\text { NCTO02862624 }\end{array}$ & vilu & $\begin{array}{l}\text { Bernhard Hering } \\
\text { Bernhard Hering }\end{array}$ & $\begin{array}{l}\text { Mimesota } \\
\text { Minnosota } \\
\end{array}$ & 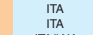 & $\begin{array}{l}8 \\
6\end{array}$ & $\begin{array}{l}\text { Completed } \\
\text { Completed }\end{array}$ & $\begin{array}{l}\text { Immunosuppresssion } \\
\text { Immunosuppression }\end{array}$ & 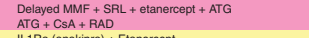 & & \\
\hline 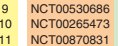 & 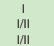 & $\begin{array}{l}\text { Marlon Levy } \\
\text { Bemhard Hering } \\
\text { Ken polonsty }\end{array}$ & $\begin{array}{l}\text { Banyor } \\
\text { Minnosta } \\
\text { Wastington } \\
\end{array}$ & $\begin{array}{c}\text { ITAMAK } \\
\text { ITA } \\
\text { ITA }\end{array}$ & $\begin{array}{l}15 \\
10 \\
8\end{array}$ & $\begin{array}{l}\text { Aative } \\
\text { Active } \\
\text { Active }\end{array}$ & $\begin{array}{l}\text { Engraftment } \\
\text { Immunosuppression } \\
\text { Metaboile }\end{array}$ & 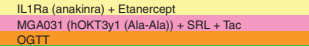 & & \\
\hline $\begin{array}{l}\text { NCT Toor888682 } \\
\text { NCT00306098 }\end{array}$ & IIII & 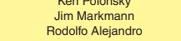 & 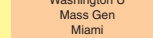 & 传 & 8 & 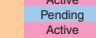 & 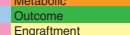 & & & Yes \\
\hline $\begin{array}{l}\text { NCT To0315614 } \\
\text { NCT To0021801 }\end{array}$ & $\|$ & 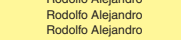 & 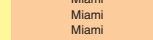 & IT & 6 & $\begin{array}{c}\substack{\text { Aative } \\
\text { Comived }} \\
\text { Compled }\end{array}$ & 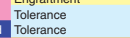 & 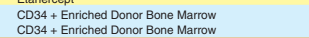 & & \\
\hline 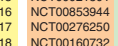 & IIII & 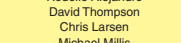 & $\begin{array}{l}\text { Vancouver } \\
\text { Emory }\end{array}$ & ITA & $\begin{array}{l}12 \\
20\end{array}$ & $\begin{array}{l}\text { Active } \\
\text { Active }\end{array}$ & $\begin{array}{l}\text { Metabolic } \\
\text { Immunosur }\end{array}$ & $\begin{array}{l}\text { Sitagiptin (DPP-4i) } \\
\text { Abatacept (CTLA4-19) }\end{array}$ & Yes & \\
\hline 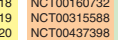 & III & 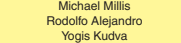 & 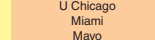 & ITA & $\begin{array}{l}50 \\
20 \\
5\end{array}$ & 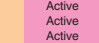 & $\begin{array}{l}\text { Outcome } \\
\text { Otucome } \\
\text { Outcomene }\end{array}$ & & & Yes \\
\hline $\begin{array}{l}\text { NCT To0315627 } \\
\text { NCTroos6817 }\end{array}$ & 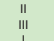 & 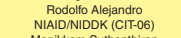 & $\begin{array}{c}\text { Mami } \\
\text { Cit.06 }\end{array}$ & ITA & $\begin{array}{l}12 \\
130\end{array}$ & 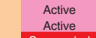 & $\begin{array}{l}\text { IImmunosuppression } \\
\text { Outcome - }\end{array}$ & 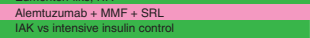 & Yes & Yes \\
\hline 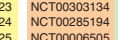 & $\|$ & $\begin{array}{l}\text { Manikkam Suthanathiran } \\
\text { Bernhard Hering } \\
\text { David Hartang }\end{array}$ & 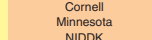 & $\begin{array}{l}\text { ITA } \\
\text { ITA } \\
\text { ITA }\end{array}$ & $\begin{array}{r}8 \\
6 \\
20\end{array}$ & $\begin{array}{l}\text { Suspended } \\
\text { Coompleted } \\
\text { Completed }\end{array}$ & $\begin{array}{l}\text { Outcome } \\
\text { Immunosuppression }\end{array}$ & 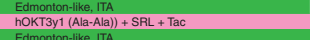 & & \\
\hline $\begin{array}{l}\text { NCT To0175256 } \\
\text { NCT To0175253 }\end{array}$ & & $\begin{array}{l}\text { Javed aranio } \\
\text { James shapio } \\
\text { James Shapiro }\end{array}$ & $\begin{array}{l}\text { Ediobonton } \\
\text { Edmonton }\end{array}$ & $\begin{array}{l}\text { ITA } \\
\text { ITA } \\
\text { ITA }\end{array}$ & $\begin{array}{l}20 \\
12 \\
12\end{array}$ & $\begin{array}{l}\text { compleced } \\
\text { complet } \\
\text { Completeded }\end{array}$ & $\begin{array}{l}\text { Ouctcome } \\
\text { Imunospression } \\
\text { Immunosuppression }\end{array}$ & 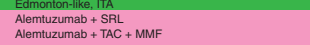 & & \\
\hline 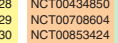 & III & 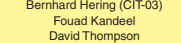 & 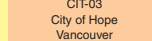 & ITA & $\begin{array}{l}20 \\
14 \\
40\end{array}$ & $\begin{array}{l}\text { Active } \\
\text { Active } \\
\text { Active }\end{array}$ & $\begin{array}{l}\text { IImmunous } \\
\text { Outcome } \\
\text { Oticomen }\end{array}$ & 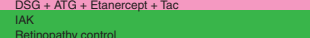 & Yes & Yes \\
\hline 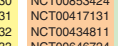 & III & 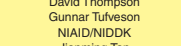 & 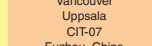 & tAK & $\begin{array}{l}20 \\
20 \\
48\end{array}$ & $\begin{array}{l}\text { Active } \\
\text { Active } \\
\text { Active }\end{array}$ & 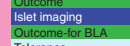 & 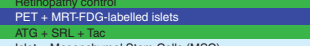 & & Yes \\
\hline 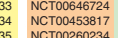 & IIII & $\begin{array}{l}\text { Jalammin Tan } \\
\text { Chrsitian Toso } \\
\text { Tos }\end{array}$ & $\begin{array}{l}\text { Fuzhou, hrina } \\
\text { Geneva }\end{array}$ & ITA & $\begin{array}{l}30 \\
15\end{array}$ & ctive & $\begin{array}{l}\text { Trelerance } \\
\text { Istetimagin }\end{array}$ & 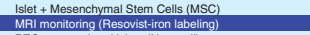 & & \\
\hline $\begin{array}{l}\text { NCTTo26260234 } \\
\text { NCT To3039231 }\end{array}$ & $\begin{array}{l}\text { IIII } \\
\text { IIII } \\
\text { III }\end{array}$ & 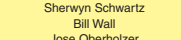 & $\begin{array}{l}\text { San Antotion Tx } \\
\text { London ont }\end{array}$ & ITA & ${ }_{8}^{12}$ & $\begin{array}{ll}\text { uspenceded } \\
\text { Pending }\end{array}$ & $\begin{array}{l}\text { Tolerance } \\
\text { Onturome }\end{array}$ & 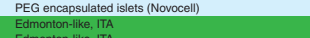 & & \\
\hline 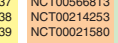 & (1111 & $\begin{array}{l}\text { Jose ondozize } \\
\text { Jon Odoric } \\
\text { NCRR }\end{array}$ & $\begin{array}{l}\text { Maddison, WI } \\
\text { NCRR }\end{array}$ & $\begin{array}{l}\text { TIA } \\
\text { TAK } \\
\text { IAK }\end{array}$ & $\begin{array}{l}10 \\
16 \\
10\end{array}$ & 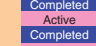 & $\begin{array}{l}\text { Outome } \\
\text { Metabolic } \\
\text { Outuonem }\end{array}$ & 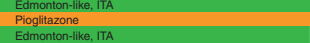 & & \\
\hline 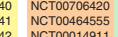 & i" & $\begin{array}{l}\text { Fouadd andeel } \\
\text { Camillo Ricordi (CIT-02) }\end{array}$ & $\begin{array}{l}\text { Cily of Hope } \\
\text { Critoro }\end{array}$ & (1) & $\begin{array}{l}20 \\
24\end{array}$ & & $\begin{array}{l}\text { Outcome } \\
\text { Metabolic }\end{array}$ & 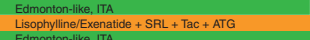 & Yes & Yes \\
\hline 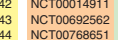 & in & $\begin{array}{l}\text { nes Shapipo (NIII01) } \\
\text { Jianming Tan } \\
\text { Peter SSnior }\end{array}$ & 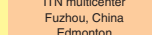 & 作 & 40 & noleted & $\begin{array}{l}\text { Outcome } \\
\text { Outome } \\
\text { Mteabolic }\end{array}$ & & & \\
\hline $\begin{array}{l}\text { NCT To0468842 } \\
\text { NCTO2028997 }\end{array}$ & IIII & $\begin{array}{l}\text { Ail Nail (CIT-05) } \\
\text { Marrtra a avakis }\end{array}$ & 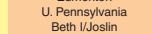 & ITA & $\begin{array}{l}12 \\
40\end{array}$ & Acive & $\begin{array}{l}\text { Tolerance } \\
\text { Oturome }\end{array}$ & $\begin{array}{l}\text { ATG }+ \text { Rituximab } \\
\text { IAK }\end{array}$ & & Yes \\
\hline 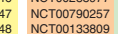 & $\|$ & $\begin{array}{l}\text { Denis Dutrane } \\
\text { Denis } \\
\text { Chis arsen }\end{array}$ & 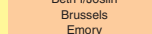 & 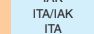 & $\begin{array}{l}15 \\
20 \\
20\end{array}$ & $\begin{array}{l}\text { Active } \\
\text { Combled } \\
\text { Comber }\end{array}$ & $\begin{array}{l}\text { Tolerance } \\
\text { SOluome } \\
\text { Otus }\end{array}$ & $\begin{array}{l}\text { Monolayer Cellular Device, SQ } \\
\text { Edmontontile IIA }\end{array}$ & & \\
\hline $\begin{array}{l}\text { NCTO0021788 } \\
\text { NCTTO0468403 }\end{array}$ & i" & $\begin{array}{l}\text { Rodolfo Alejeandro } \\
\text { Shapiriol Larsen (CIT-04) }\end{array}$ & 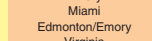 & ITA & $\begin{array}{l}20 \\
20\end{array}$ & $\begin{array}{l}\text { Completed } \\
\text { Active }\end{array}$ & $\begin{array}{l}\text { Outcome } \\
\text { Immunosu }\end{array}$ & $\begin{array}{l}\text { Edmonton-1ilie, ITA } \\
\text { Belatacept }+ \text { Simulect + MMF }\end{array}$ & Yes & Yes \\
\hline 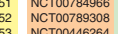 & if & $\begin{array}{l}\text { OAdran Coterel } \\
\text { Olle Korsgren (CIT-01A) }\end{array}$ & $\begin{array}{l}\text { Nordichana Network } \\
\text { Nerk }\end{array}$ & IAK & $\begin{array}{l}10 \\
36 \\
36\end{array}$ & $\begin{array}{l}\text { Pendivg } \\
\text { Active }\end{array}$ & $\begin{array}{l}\text { Ouccome } \\
\text { Engraftment }\end{array}$ & 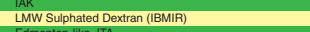 & Yes & Yes \\
\hline 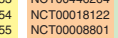 & vil & $\begin{array}{l}\text { Francolis pattou } \\
\text { Nermard Hering } \\
\text { Bernard }\end{array}$ & $\begin{array}{l}\text { Mile } \\
\text { NCRR } \\
\text { NCPR }\end{array}$ & . & $\begin{array}{c}14 \\
15 \\
10\end{array}$ & 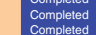 & 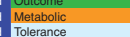 & 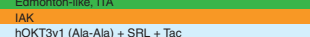 & & \\
\hline 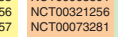 & & $\begin{array}{l}\text { Pierre eanhamo } \\
\text { Mark tharyy }\end{array}$ & $\begin{array}{l}\text { GRAGl Networ: } \\
\text { Columbia }\end{array}$ & ITA & $\begin{array}{l}22 \\
10\end{array}$ & 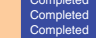 & 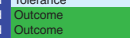 & & & \\
\hline $\begin{array}{l}\text { NCTT000409461 } \\
\text { NCTOO790439 }\end{array}$ & $\|$ & $\begin{array}{l}\text { Bart Keymeulen } \\
\text { Olle Rorstren (CIT-1B) }\end{array}$ & $\begin{array}{l}\text { Belgium } \\
\text { Novric Networt }\end{array}$ & ITA & $\begin{array}{l}10 \\
36\end{array}$ & & Engrattmen & (BMIRT) & Yes & \\
\hline 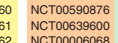 & in & $\begin{array}{l}\text { Robert Sherwin } \\
\text { Pierre Benhamou }\end{array}$ & $\begin{array}{l}\text { Yale } \\
\text { Grenoble } \\
\text { enole }\end{array}$ & ITA & $\begin{array}{l}36 \\
21\end{array}$ & tivive & $\begin{array}{l}\text { Outcome } \\
\text { Outuome } \\
\text { neme }\end{array}$ & & & \\
\hline 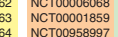 & " & 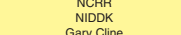 & 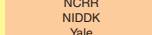 & & ${ }_{20}^{20} \mathrm{Y}-\mathrm{C}$ & 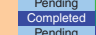 & 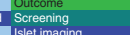 & PET & & \\
\hline 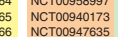 & IIIII & $\begin{array}{l}\text { Garanc Cine } \\
\text { Joker } \\
\text { Mohan }\end{array}$ & Living Cell Tecechnolog & TIA & 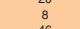 & & 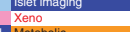 & 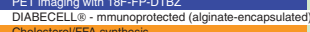 & & \\
\hline 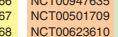 & ini & 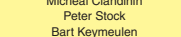 & 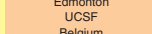 & 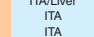 & 46 & 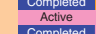 & 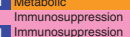 & 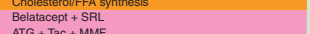 & & \\
\hline 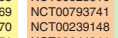 & & 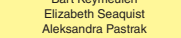 & $\begin{array}{c}\text { Mine } \\
\text { Transitio Th }\end{array}$ & ITAM & 20 & mpleted & 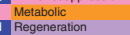 & 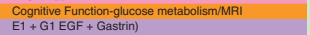 & Yes & \\
\hline 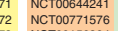 & "I & $\begin{array}{l}\text { Ani Bnhanali } \\
\text { Paul haris }\end{array}$ & $\begin{array}{l}\text { handigarhb India } \\
\text { Columbia U }\end{array}$ & 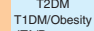 & $\begin{array}{l}100 \\
30\end{array}$ & & $\begin{array}{l}\text { Regeneratan } \\
\text { Ilist imaging }\end{array}$ & 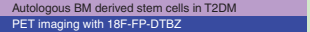 & & \\
\hline 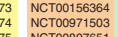 & & $\begin{array}{l}\text { Michael Muar } \\
\text { Aleiantro Mesples }\end{array}$ & 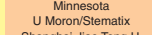 & T1DM & ${ }^{34}$ & & $\begin{array}{l}\text { Outcome } \\
\text { Regeneration }\end{array}$ & $\begin{array}{l}\text { Renal protection } \\
\text { Autrologous BM arth }\end{array}$ & & \\
\hline 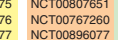 & & 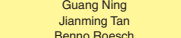 & 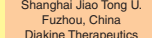 & $\begin{array}{l}T 100 \\
T \\
T\end{array}$ & & & $\begin{array}{l}\text { Regeneraton } \\
\text { Regention } \\
\text { Regenartion }\end{array}$ & 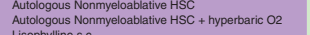 & & \\
\hline $\begin{array}{l}\text { Pending } \\
\text { NCTroogo066 }\end{array}$ & IIII & 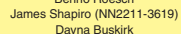 & 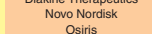 & (T) & $\begin{array}{l}0 \\
40 \\
60\end{array}$ & nang & 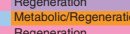 & 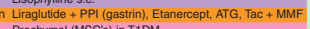 & . & \\
\hline & & 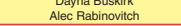 & Xx Falls, S. Dakota & & & & $\begin{array}{l}\text { Regeneration } \\
\text { Regeneration }\end{array}$ & 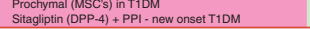 & $\begin{array}{l}\text { Yes } \\
\text { Yes }\end{array}$ & \\
\hline
\end{tabular}

Abbreviations: ITA, islet transplant alone; IAK, islet after kidney; CIT, Clinical Islet Transplant Consortium; IBMIR, instant blood-mediated inflammatory reaction; MMF, mycophenolate mofetil; SRL, sirolimus; ATG, antithymocyte globulin; CsA, cyclosporine; Tac, tacrolimus; BLA, biological licensure; Thymo, thymoglobulin; DSG, deoxyspergualin; RCT, randomized controlled trial.

selected indications. Similarly, the National Institute for Health and Clinical Excellence (NICE) in the United Kingdom recently fully funded islet transplantation, and several other countries including Sweden, Switzerland, France, and Italy have followed suit. "Brittle" type 1 diabetes with associated recurrent hypoglycemic episodes or marked glycemic lability, despite best efforts by a qualified diabetologist or endocrinologist to stabilize control with optimized insulin delivery including intensive insulin, in- sulin pumps, or continuous glucose monitoring systems (CGMS), currently form the basis for consideration of islet-alone transplantation. The major islet transplant centers involved with the Clinical Islet Transplant Consortium (CIT) currently use a Clarke Score $>4$, a Hypo Score $\geq 1000$, a Lability Index (LI) score $\geq 400 \mathrm{~mm} / \mathrm{L}^{2} / \mathrm{h} \cdot \mathrm{wk}^{-1}$, or a combination of Hypo Score $\geq 400$ and LI $\geq 300$, as threshold indications for enrollment in islet-alone transplant trials. 


\section{Islet Engraftment}

The initial few days following islet infusion are a critical time. It is clear that a large proportion of the graft is lost during this period, up to $60 \%$ in both syngeneic mouse islet models (Biarnes et al. 2002) and clinically in the allogeneic setting (Eich et al. 2007b). Below we will review approaches to enhancing engraftment, which may improve islet survival and function leading to improved single-donor islet transplant success.

\section{Anti-Inflammatory}

When islets become trapped in portal venules they are relatively ischemic and inflamed before undergoing revascularization, aided by the recipient arterial system. This places islets at direct risk of undergoing apoptosis and necrosis. More specifically, islets are subject to attack by a number of cytokines, including tumor necrosis factor $\alpha$ (TNF- $\alpha)$ and Interleukin 1 (IL-1).

An initial study by Farney et al. (1993) showed the benefit of TNF- $\alpha$ blockade in mice receiving syngeneic islet grafts. This led to the inclusion of etanercept, a fusion protein that includes the TNF receptor, in a single-donor islet transplant protocol reported by Hering et al. (2005). Eight patients received an average of 7271 IEQ/ kg from an optimal donor pancreas in addition to etanercept (50 $\mathrm{mg}$ IV day 0 and $25 \mathrm{mg}$ on days 3, 7, and 10) antithymocyte globulin (ATG) and daclizumab for induction therapy. Islets were cultured for a period of 2 days before transplant. All eight patients became insulin independent (Hering et al. 2005). It remains unclear at this time how much the period of culture and anti-inflammatory strategies contributed to single-donor islet engraftment. Etanercept has now been included in many other islet transplant protocols including the University of Chicago (Gangemi et al. 2008), the University of Miami (Faradji et al. 2008), and the University of Alberta. Alternative TNF- $\alpha$ blockade with the monoclonal antibody Infliximab, although potent inflammatory disorders including inflammatory bowel disease, has not shown a benefit in islet transplantation (Froud et al. 2005). It is difficult to explain why etaner- cept may be more potent than Infliximab for suppression of TNF- $\alpha$ during islet engraftment, or indeed whether multiple other differences in the islet product and engraftment regimens outside of TNF- $\alpha$ contributed to single-donor engraftment success.

Blockade of IL-1 with anakinra, an IL-1 receptor antagonist, has become important in the treatment of inflammatory conditions including rheumatoid arthritis (Mertens and Singh 2009). Matsumoto et al. (2011) published results of three patients who received a combination of etanercept and anakinra, reporting benefits to early graft function. Our own laboratory has investigated the combination of TNF $\alpha /$ IL- 1 blockade and found them to be synergistic in promoting islet engraftment in mice (McCall et al. 2011a).

\section{Apoptosis and IBMIR}

The team in Uppsala, Sweden led by Dr. Olle Korsgren has extensively studied the effects of IBMIR and its negative effect on engrafting islets (Bennet et al. 2000; Nilsson et al. 2011). IBMIR involves a thrombotic and inflammatory reaction characterized by binding of platelets to the islet surface and infiltration of leukocytes in the islets. This is likely mediated by tissue factor expressed on the islets themselves. The group has developed multiple potential targets to reduce the effects of IBMIR including dextran sulfate and islet surface heparinization (Johansson et al. 2006; Cabric et al. 2007). Intravenous infusions of heparin and insulin posttransplant have been reported to improve the success of single-donor islet transplantation (Koh et al. 2010).

Apoptosis, a caspase-mediated event, is a dominant final pathway for early islet death. Methods to prevent islet apoptosis in the early posttransplant period have been extensively studied by our laboratory and others. Emamaullee et al. engineered rodent islets to overexpress X-linked inhibitor of apoptosis protein (XIAP) (Emamaullee et al. 2005). This rendered them relatively resistant to apoptosis. Although modification of islets with viral vectors may not be palatable in the clinical setting, this "proof of principle" inspired studies exploring caspase 
inhibitors. We have recently studied the compound IDN6556 (Conatus Pharmaceuticals, Inc., San Diego, CA) in both mouse and pig islet transplant models (McCall et al. 2011b). In both cases, addition of the drug posttransplant led to improvements in marginal mass islet engraftment. In other words, a minimal islet graft size was able to ameliorate diabetes in mice when treated with the caspase inhibitor. A further compound (EP1013) was tested that showed the ability to promote allograft survival when combined with costimulation blockade. A clinical trial is planned with IDN6556 and if the results mirror those seen in the preclinical studies this approach could substantially reduce the islet mass necessary to secure insulin independence and the antigenic burden placed on the immune system by dying islets.

\section{Metabolic and Growth Stimulation}

Glucagonlike-peptide 1 (GLP1) is an endogenous peptide secreted form intestinal $L$ cells that functions to increase insulin secretion and $\beta$ cell mass. Analogs of GLP1 are currently used in the management of type 2 diabetes and have shown promise in the setting of single-donor islet transplantation. The ability of exenatide to aid islet engraftment has been shown by multiple centers including the universities of Illinois, Miami, and British Columbia (Ghofaili et al. 2007; Faradji et al. 2008; Gangemi et al. 2008). However, $30 \%$ of patients on exenatide develop intolerable nausea. An alternative compound, liraglutide, has been shown to possess similar effects, benefitting human islet culture and engraftment in mice (Merani et al. 2008b; Toso et al. 2010). This compound seems to be better tolerated in patients.

\section{Advances in Recipient Immunosuppression}

Effective control of both alloimmune and autoimmune islet attack in the recipient remains the cornerstone of posttransplant islet recipient treatment. Unfortunately, many of the compounds used are toxic to the islets themselves. This is especially true of corticosteroids that formed the backbone of islet transplant immu- nosuppression before the Edmonton Protocol (Morel et al. 1992; Shapiro et al. 1998). One of the main strengths of the Edmonton Protocol report was its avoidance of corticosteroid use. In its place, sirolimus (a macrolide antibiotic which inhibits mTOR) and tacrolimus (Tac) (a calcineurin inhibitor) were used (Shapiro et al. 2000). These are not without their own side effects as tacrolimus can cause neuro- and nephrotoxicity in addition to $\beta$-cell damage (Froud et al. 2006; Chatenoud 2008). The choice to eliminate tacrolimus and/or sirolimus may improve metabolic function but may be detrimental when considering the risk of rejection. Mycophenolate mofetil (MMF, CellCept), a purine biosynthesis inhibitor, is now more commonly used either in combination with tacrolimus or sirolimus (Table 2).

Induction therapy is used in most islet transplant protocols. The Edmonton Protocol used daclizumab, an anti-interleukin 2 receptor monoclonal antibody (anti-CD25) given pretransplant and for four doses biweekly posttransplant (Shapiro et al. 2000). Daclizumab is now off the market as its patent has expired. The Minnesota program reported use of an antiT-cell strategy with anti-CD3 (hOKT3-ala-ala) which, when combined with sirolimus and lowdose tacrolimus, promoted single-donor insulin independence in four of six recipients (Hering et al. 2004). More recently at the University of Alberta, we have noted marked and sustained improvement in 5-yr insulin independence outcomes that now match the results of pancreasalone transplantation at a similar time point, when alemtuzumab (anti-CD52) T-depletional induction is given. The combination of alemtuzumab induction with tacrolimus and MMF maintenance has led to durable protection against both auto- and alloimmune pathways. Despite this approach, single-donor islet engraftment success at the University of Alberta has remained at approximately $10 \%$. This reflects our less selective approach both to pancreas donors and recipients, and emphasizes the need to address early engraft and peritransplant survival of portally infused cells.

A number of exciting new immunosuppressive agents are emerging that may provide 
Update on Islet Transplantation

Table 2. Summary of current major clinical trials in islet transplantation, with single-donor success rate and induction protocol

\begin{tabular}{|c|c|c|c|c|c|}
\hline Center & $\begin{array}{c}\text { Year of } \\
\text { publication }\end{array}$ & Induction & Maintenance therapy & $\begin{array}{c}\text { Number } \\
\text { of donors }^{\mathrm{a}}\end{array}$ & $\begin{array}{c}\text { Insulin- } \\
\text { independence } \\
\text { rate }^{\mathrm{b}} \\
\end{array}$ \\
\hline Alberta & 2000 & Daclizumab & Sirolimus, Tacrolimus & $2-4$ & $7 / 7(100 \%)$ \\
\hline Minnesota & 2004 & Anti-CD3, Sirolimus & Sirolimus, Tacrolimus & 1 & $4 / 6(67 \%)$ \\
\hline Miami & 2005 & $\begin{array}{l}\text { Daclizumab } \\
\text { Infliximab, } \\
\text { Daclizumab }\end{array}$ & $\begin{array}{l}\text { Sirolimus, Tacrolimus } \\
\text { Sirolimus, Tacrolimus }\end{array}$ & $\begin{array}{l}1-2 \\
1-2\end{array}$ & $\begin{array}{l}7 / 8(88 \%) \\
7 / 8(88 \%)\end{array}$ \\
\hline Minnesota & 2005 & $\begin{array}{l}\text { ATG, Daclizumab, } \\
\text { Etanercept }\end{array}$ & MMF, Sirolimus, Tacrolimus & 1 & $8 / 8(100 \%)$ \\
\hline $\begin{array}{l}\text { Chicago, } \\
\text { Illinois }\end{array}$ & 2008 & $\begin{array}{l}\text { Daclizumab } \\
\text { Etanercept, } \\
\text { Exenatide, } \\
\text { Daclizumab }\end{array}$ & $\begin{array}{l}\text { Sirolimus, Tacrolimus } \\
\text { Sirolimus, Tacrolimus }\end{array}$ & $\begin{array}{c}2-3 \\
1\end{array}$ & $\begin{array}{l}4 / 4(100 \%) \\
\quad 6 / 6(100 \%)\end{array}$ \\
\hline San Francisco & 2010 & ATG & $\begin{array}{l}\text { Efalizumab and Sirolimus or } \\
\text { MMF }\end{array}$ & $1-2$ & $8 / 8(100 \%)$ \\
\hline San Francisco & 2010 & ATG & $\begin{array}{l}\text { Sirolimus or MMF and } \\
\text { Belatacept or Efalizumab }\end{array}$ & $\begin{array}{l}1 \\
1\end{array}$ & $\begin{array}{l}4 / 5(80 \%) \\
3 / 5(60 \%)\end{array}$ \\
\hline $\begin{array}{l}\text { Baylor } \\
\text { (Houston) }\end{array}$ & 2011 & $\begin{array}{l}\text { Daclizumab } \\
\text { ATG, Etanercept }\end{array}$ & $\begin{array}{l}\text { Sirolimus, Tacrolimus } \\
\text { Tacrolimus, MMF, } \\
\text { Anakinra }\end{array}$ & $\begin{array}{l}2 \\
1\end{array}$ & $\begin{array}{l}3 / 3(100 \%) \\
\quad 3 / 3(100 \%)\end{array}$ \\
\hline
\end{tabular}

${ }^{\mathrm{a}}$ Number of pancreas donors per recipient.

${ }^{\mathrm{b}}$ Rate of achievement of insulin independence.

Abbreviation: ATG, antithymocyte globulin.

superior immunosuppression with elimination of existing agents such as tacrolimus. The combination of sirolimus and MMF is one option but this may not provide potent long-term immunosuppression to prevent rejection. Costimulation blockade has seen increasing use in organ transplantation clinical trials (Bluestone 2005; Vincenti et al. 2005). Two compounds have currently been approved for use: abatacept (CTLA4Ig) and belatacept (LEA29Y), which block T-cell interaction with CD80/ CD86 on antigen-presenting cells. The group at UCSF has reported promising results with belatacept costimulation blockade in combination with thymoglobulin (thymo)-based therapy (Posselt et al. 2010b). This protocol allowed a calcineurin-inhibitor-free maintenance therapy strategy and even permitted single-donor engraftment success. This same group has studied the benefit of an anti-LFA-1 antibody called efalizumab (Posselt et al. 2010a,b). Although effective in allowing tacrolimus-free maintenance therapy, it has been withdrawn from clinical use owing to concerns of progressive multifocal leukoencephalopathy and thus the long-term followup in these studies was hampered.

\section{Advances in Immune Monitoring and Control of Recurrent Autoimmunity}

Although islet transplantation is currently the most minimally invasive transplant available (perhaps with the exception of a blood transfusion), and is attractive from a conceptual perspective, its relatively tiny cellular mass distributed diffusely throughout the liver creates especially unique challenges for detection, imaging, and monitoring post transplant. The inability to detect early islet rejection at a time point where reversal is still possible, combined with a need to control recurrence of autoimmunity in an already highly primed subject with type 1 diabetes, conversely create one of the most difficult transplants to maintain. Lessons 
learned previously in whole pancreas transplantation suggested that minimal maintenance immunosuppression would probably control autoimmunity, but it is now becoming increasingly apparent that even in whole pancreas transplantation complete autoimmune-mediated destruction leads to graft loss in at least $6 \%-10 \%$ (and maybe more) over time (Vendrame et al. 2010; Burke et al. 2011; Pugliese et al. 2011). Clear histological evidence of recurrent autoimmunity with selective $\beta$-cell destruction after islet transplantation has been shown by Rossini's group (Rossini 2004; Sharma et al. 2006). Although core biopsy of an intrahepatic islet transplant is technically possible, the approach is unattractive to patients, and only yields islet material in about half of the cases (Toso et al. 2009). However, encouraging data is beginning to emerge from sequential analysis of peripheral blood. Perturbations in autoantibody levels in peripheral blood for islet cell autoantibody (ICA-512), glutamic acid decarboxylase (GAD-65), and anti-insulin antibody (IA-2) over time have generally not yielded distinctive patterns predictive of eventual islet outcome, possibly in part owing to imprecision in the monitoring of these antibodies, and perhaps suggesting that the presence of such antibodies could be epiphenomena, and not always predictive of potency of autoreactive memory responses. Conversely, Roep's group have found striking correlations between cellular autoreactivity in lymphocyte stimulation tests against autoantigens and alloreactivity using cytotoxic T-cell lymphocyte precursor assays and outcome in clinical islet transplantation (Huurman et al. 2008). Furthermore, the same group found a strong association between cytokine profiles in mixed lymphocyte culture (MLC) assays using peripheral blood mononuclear cells and graftspecific HLA class II. They also measured cytotoxic T-lymphocyte precursor (CTLp) assays to determine HLA class I alloreactivity, and found that a marked skew toward a Th2 or regulatory phenotype in those subjects maintaining insulin independence over time, with production of IL10 and low-MLC reactivity strongly associated with persistence insulin independence over time (Huurman et al. 2009).
Kenyon's group in Miami have studied perturbations in granzyme $\mathrm{B}$ and perforin messenger RNA (mRNA) expression within cytotoxic lymphocytes, and found that especially granzyme B mRNA expression can be predictive of eventual islet failure, and may occur up to many months before the onset of hyperglycemia (Han et al. 2002, 2004). Although these observations are enticing, until the course of inevitable graft failure is clearly averted by interventions dictated by elevated granzyme B expression, their utility will remain unclear.

New technologies for the study of peptideMHC T-cell ligands using tetramer-based approaches have been developed by Nepom's group, and allow more precise visualization of autoreactive T-lymphocyte responses (Nepom 2005). Such approaches may further predict which subjects may do better in the longer term with an islet transplant, and may further help to direct T-cell-specific immunotherapies for control of recurrent autoimmunity post transplant. Until such time as "personalized medicine"-based approaches are developed and applied in the setting of clinical islet transplantation, highly potent T-direct inductive therapy and increased dosing of maintenance immunosuppression will be required in all cases, if recurrent autoimmunity and protection from allograft rejection is to be controlled effectively.

\section{CONCLUDING REMARKS}

There have been substantial advances in clinical islet transplantation over the past 12 years. Before this time, insulin independence was an extremely rare occurrence. The Edmonton Protocol success invigorated the field and prompted continued research and effort into improving islet transplant outcomes. Although the initial results of this series were promising using multiple islet donors, the insulin-independence rates declined markedly by 3 and 5 years after islet infusion. In the years following, groups have improved on these results; now single islet donors can provide insulin independence. The routine achievement of these results will allow for many more subjects to be treated with islets and will reduce risk of donor HLA sensitization. 
In addition, insulin-independence rates using multiple islet donors are nearing those for pancreas transplantation making decisions around pancreas allocation less straightforward. Advances in engraftment and immunosuppressive agents have permitted these improved results. How can we further advance islet transplantation so that single-donor insulin independence is routine? This will require a multimodal approach including the optimization of the donor pancreas, access to consistent and effective collagenase enzyme blends, improved islet engraftment and immunosuppressive agents, and further advances in islet graft monitoring. This will no doubt improve both short- and long-term islet transplantation success and further open up this exciting treatment to diabetic patients.

\section{ACKNOWLEDGMENTS}

Funding for the clinical islet transplant program comes from the Clinical Islet Transplant Consortium (CIT) from the National Institute of Diabetes and Digestive Diseases (NIDDK) and the National Institute for Allergy, Immunology and Diabetes (NIAID) from the National Institutes of Health (NIH), from a program grant from the Juvenile Diabetes Research Foundation (JDRF) and from the Diabetes Research Institute Foundation of Canada (DRIFCan). A.M.J.S. is supported through a senior clinical scholarship from Alberta Innovates Healthcare Solutions (AIHS). M.M. is the recipient of a clinical fellowship from AIHS.

\section{REFERENCES}

Ballinger WF, Lacy PE. 1972. Transplantation of intact pancreatic islets in rats. Surgery 72: 175-186.

Bennet W, Sundberg B, Groth CG, Brendel MD, Brandhorst D, Brandhorst H, Bretzel RG, Elgue G, Larsson R, Nilsson B, et al. 1999. Incompatibility between human blood and isolated islets of Langerhans: A finding with implications for clinical intraportal islet transplantation? Diabetes 48: 1907-1914.

Bennet W, Groth CG, Larsson R, Nilsson B, Korsgren O. 2000. Isolated human islets trigger an instant blood mediated inflammatory reaction: Implications for intraportal islet transplantation as a treatment for patients with type 1 diabetes. Ups J Med Sci 105: 125-133.

Berney T, Ferrari-Lacraz S, Buhler L, Oberholzer J, Marangon N, Philippe J, Villard J, Morel P. 2009. Long-term insulin-independence after allogeneic islet transplantation for type 1 diabetes: Over the 10-year mark. Am J Transplant 9: 419-423.

Biarnes M, Montolio M, Nacher V, Raurell M, Soler J, Montanya E. 2002. $\beta$-cell death and mass in syngeneically transplanted islets exposed to short- and long-term hyperglycemia. Diabetes 51: 66-72.

Bluestone JA. 2005. CTLA-4Ig is finally making it: A personal perspective. Am J Transplant 5: 423-424.

Brandhorst H, Friberg A, Nilsson B, Andersson HH, Felldin M, Foss A, Salmela K, Tibell A, Tufveson G, Korsgren O, et al. 2010. Large-scale comparison of Liberase HI and collagenase NB1 utilized for human islet isolation. Cell Transplant 19: 3-8.

Burke GW III, Vendrame F, Pileggi A, Ciancio G, Reijonen H, Pugliese A. 2011. Recurrence of autoimmunity following pancreas transplantation. Curr Diab Rep 11: 413-419.

Cabric S, Sanchez J, Lundgren T, Foss A, Felldin M, Kallen R, Salmela K, Tibell A, Tufveson G, Larsson R, et al. 2007. Islet surface heparinization prevents the instant bloodmediated inflammatory reaction in islet transplantation. Diabetes 56: 2008-2015.

Calafiore R, Basta G, Luca G, Lemmi A, Montanucci MP, Calabrese G, Racanicchi L, Mancuso F, Brunetti P. 2006 Microencapsulated pancreatic islet allografts into nonimmunosuppressed patients with type 1 diabetes: First two cases. Diabetes Care 29: 137-138.

Cantarelli E, Melzi R, Mercalli A, Sordi V, Ferrari G, Lederer CW, Mrak E, Rubinacci A, Ponzoni M, Sitia G, et al. 2009. Bone marrow as an alternative site for islet transplantation. Blood 114: 4566-4574.

Chatenoud L. 2008. Chemical immunosuppression in islet transplantation-Friend or foe? N Engl J Med 358: 1192 1193.

Chick WL, Perna JJ, Lauris V, Low D, Galletti PM, Panol G, Whittemore AD, Like AA, Colton CK, Lysaght MJ. 1977. Artificial pancreas using living $\beta$ cells: Effects on glucose homeostasis in diabetic rats. Science 197: 780-782.

Christoffersson G, Henriksnas J, Johansson L, Rolny C, Ahlstrom H, Caballero-Corbalan J, Segersvard R, Permert J, Korsgren O, Carlsson PO, et al. 2010. Clinical and experimental pancreatic islet transplantation to striated muscle: Establishment of a vascular system similar to that in native islets. Diabetes 59: 2569-2578.

Duvivier-Kali VF, Omer A, Parent RJ, O’Neil JJ, Weir GC. 2001. Complete protection of islets against allorejection and autoimmunity by a simple barium-alginate membrane. Diabetes 50: 1698-1705.

Duvivier-Kali VF, Omer A, Lopez-Avalos MD, O’Neil JJ, Weir GC. 2004. Survival of microencapsulated adult pig islets in mice in spite of an antibody response. Am J Transplant 4: 1991-2000.

Echeverri GJ, McGrath K, Bottino R, Hara H, Dons EM, van der Windt DJ, Ekser B, Casu A, Houser S, Ezzelarab M, et al. 2009. Endoscopic gastric submucosal transplantation of islets (ENDO-STI): Technique and initial results in diabetic pigs. Am J Transplant 9: 2485-2496.

Eich T, Eriksson O, Lundgren T. 2007a. Visualization of early engraftment in clinical islet transplantation by positronemission tomography. N Engl J Med 356: 2754-2755. 
Eich T, Eriksson O, Sundin A, Estrada S, Brandhorst D, Brandhorst H, Langstrom B, Nilsson B, Korsgren O, Lundgren T. 2007b. Positron emission tomography: A real-time tool to quantify early islet engraftment in a preclinical large animal model. Transplantation 84: 893-898.

Elliott RB, Escobar L, Tan PL, Muzina M, Zwain S, Buchanan C. 2007. Live encapsulated porcine islets from a type 1 diabetic patient $9.5 \mathrm{yr}$ after xenotransplantation. Xenotransplantation 14: 157-161.

Emamaullee JA, Rajotte RV, Liston P, Korneluk RG, Lakey JR, Shapiro AM, Elliott JF. 2005. XIAP overexpression in human islets prevents early posttransplant apoptosis and reduces the islet mass needed to treat diabetes. Diabetes 54: $2541-2548$.

Eriksson O, Alavi A. 2011. Imaging the islet graft by positron emission tomography. Eur J Nucl Med Mol Imaging 39: 533-542.

Eriksson O, Eich T, Sundin A, Tibell A, Tufveson G, Andersson H, Felldin M, Foss A, Kyllonen L, Langstrom B, et al. 2009. Positron emission tomography in clinical islet transplantation. Am J Transplant 9: 2816-2824.

Faradji RN, Tharavanij T, Messinger S, Froud T, Pileggi A, Monroy K, Mineo D, Baidal DA, Cure P, Ponte G, et al. 2008. Long-term insulin independence and improvement in insulin secretion after supplemental islet infusion under exenatide and etanercept. Transplantation 86: $1658-1665$.

Farney AC, Xenos E, Sutherland DE, Widmer M, Stephanian E, Field MJ, Kaufman DB, Stevens RB, Blazar B, Platt J, et al. 1993. Inhibition of pancreatic islet $\beta$ cell function by tumor necrosis factor is blocked by a soluble tumor necrosis factor receptor. Transplant Proc 25: 865-866.

Froud T, Ricordi C, Baidal DA, Hafiz MM, Ponte G, Cure P, Pileggi A, Poggioli R, Ichii H, Khan A, et al. 2005. Islet transplantation in type 1 diabetes mellitus using cultured islets and steroid-free immunosuppression: Miami experience. Am J Transplant 5: 2037-2046.

Froud T, Baidal DA, Ponte G, Ferreira JV, Ricordi C, Alejandro R. 2006. Resolution of neurotoxicity and $\beta$-cell toxicity in an islet transplant recipient following substitution of tacrolimus with MMF. Cell Transplant 15: 613-620.

Gangemi A, Salehi P, Hatipoglu B, Martellotto J, Barbaro B, Kuechle JB, Qi M, Wang Y, Pallan P, Owens C, et al. 2008. Islet transplantation for brittle type 1 diabetes: The UIC protocol. Am J Transplant 8: 1250-1261.

Ghofaili KA, Fung M, Ao Z, Meloche M, Shapiro RJ, Warnock GL, Elahi D, Meneilly GS, Thompson DM. 2007. Effect of exenatide on $\beta$ cell function after islet transplantation in type 1 diabetes. Transplantation 83: 24-28.

Han D, Xu X, Pastori RL, Ricordi C, Kenyon NS. 2002. Elevation of cytotoxic lymphocyte gene expression is predictive of islet allograft rejection in nonhuman primates. Diabetes 51: 562-566.

Han D, Xu X, Baidal D, Leith J, Ricordi C, Alejandro R, Kenyon NS. 2004. Assessment of cytotoxic lymphocyte gene expression in the peripheral blood of human islet allograft recipients: Elevation precedes clinical evidence of rejection. Diabetes 53: 2281-2290.

Hering BJ, Kandaswamy R, Harmon JV, Ansite JD, Clemmings SM, Sakai T, Paraskevas S, Eckman PM, Sageshima J, Nakano M, et al. 2004. Transplantation of cultured islets from two-layer preserved pancreases in type 1 diabetes with anti-CD3 antibody. Am J Transplant 4: 390-401.

Hering BJ, Kandaswamy R, Ansite JD, Eckman PM, Nakano M, Sawada T, Matsumoto I, Ihm SH, Zhang HJ, Parkey J, et al. 2005. Single-donor, marginal-dose islet transplantation in patients with type 1 diabetes. JAMA 293: 830835.

Huurman VA, Hilbrands R, Pinkse GG, Gillard P, Duinkerken G, van de Linde P, van der Meer-Prins PM, Versteeg-van der Voort Maarschalk MF, Verbeeck K, Alizadeh BZ, et al. 2008. Cellular islet autoimmunity associates with clinical outcome of islet cell transplantation. PLoS ONE 3: e2435.

Huurman VA, Velthuis JH, Hilbrands R, Tree TI, Gillard P, van der Meer-Prins PM, Duinkerken G, Pinkse GG, Keymeulen B, Roelen DL, et al. 2009. Allograft-specific cytokine profiles associate with clinical outcome after islet cell transplantation. Am J Transplant 9: 382-388.

Johansson H, Lukinius A, Moberg L, Lundgren T, Berne C, Foss A, Felldin M, Kallen R, Salmela K, Tibell A, et al. 2005. Tissue factor produced by the endocrine cells of the islets of Langerhans is associated with a negative outcome of clinical islet transplantation. Diabetes 54: 1755-1762.

Johansson H, Goto M, Dufrane D, Siegbahn A, Elgue G, Gianello P, Korsgren O, Nilsson B. 2006. Low molecular weight dextran sulfate: A strong candidate drug to block IBMIR in clinical islet transplantation. Am J Transplant 6: $305-312$.

Kin T, Johnson PR, Shapiro AM, Lakey JR. 2007. Factors influencing the collagenase digestion phase of human islet isolation. Transplantation 83: 7-12.

Kin T, Zhai X, O'Gorman D, Shapiro AM. 2008. Detrimental effect of excessive collagenase class II on human islet isolation outcome. Transpl Int 21: 1059-1065.

Koh A, Senior P, Salam A, Kin T, Imes S, Dinyari P, Malcolm A, Toso C, Nilsson B, Korsgren O, et al. 2010. Insulinheparin infusions peritransplant substantially improve single-donor clinical islet transplant success. Transplantation 89: 465-471.

Lake SP, Bassett PD, Larkins A, Revell J, Walczak K, Chamberlain J, Rumford GM, London NJ, Veitch PS, Bell PR, et al. 1989. Large-scale purification of human islets utilizing discontinuous albumin gradient on IBM 2991 cell separator. Diabetes 38: 143-145.

Markmann JF, Deng S, Desai NM, Huang X, Velidedeoglu E, Frank A, Liu C, Brayman KL, Lian MM, Wolf B, et al. 2003. The use of non-heart-beating donors for isolated pancreatic islet transplantation. Transplantation 75: $1423-1429$.

Matsumoto I, Sawada T, Nakano M, Sakai T, Liu B, Ansite JD, Zhang HJ, Kandaswamy R, Sutherland DE, Hering BJ. 2004. Improvement in islet yield from obese donors for human islet transplants. Transplantation 78: 880-885.

Matsumoto S, Okitsu T, Iwanaga Y, Noguchi H, Nagata H, Yonekawa Y, Yamada Y, Fukuda K, Tsukiyama K, Suzuki $\mathrm{H}$, et al. 2005. Insulin independence after living-donor distal pancreatectomy and islet allotransplantation. Lancet 365: $1642-1644$.

Matsumoto S, Takita M, Chaussabel D, Noguchi H, Shimoda M, Sugimoto K, Itoh T, Chujo D, Sorelle J, Onaca N, et al. 2011. Improving efficacy of clinical islet transplantation with iodixanol based islet purification, 
thymoglobulin induction and blockage of IL-1- $\beta$ and TNF- $\alpha$. Cell Transplant 20: 1641-1647.

McCall M, Pawlick R, Kin T, Shapiro AM. 2011a. Anakinra potentiates the protective effects of etanercept in transplantation of marginal mass human islets in immunodeficient mice. Am J Transplant 12: 322-329.

McCall M, Toso C, Emamaullee J, Pawlick R, Edgar R, Davis J, Maciver A, Kin T, Arch R, Shapiro AM. 2011b. The caspase inhibitor IDN-6556 (PF3491390) improves marginal mass engraftment after islet transplantation in mice. Surgery 150: 48-55.

Medarova Z, Moore A. 2009. MRI as a tool to monitor islet transplantation. Nat Rev Endocrinol 5: 444-452.

Mehigan DG, Bell WR, Zuidema GD, Eggleston JC, Cameron JL. 1980. Disseminated intravascular coagulation and portal hypertension following pancreatic islet autotransplantation. Ann Surg 191: 287-293.

Merani S, Toso C, Emamaullee J, Shapiro AMJ. 2008a. Optimal implantation site for pancreatic islet transplantation. Br J Surgery 95: 1449-1461.

Merani S, Truong W, Emamaullee JA, Toso C, Knudsen LB, Shapiro AM. 2008b. Liraglutide, a long-acting human glucagon-like peptide 1 analog, improves glucose homeostasis in marginal mass islet transplantation in mice. Endocrinology 149: 4322-4328.

Mertens M, Singh JA. 2009. Anakinra for rheumatoid arthritis. Cochrane Database Syst Rev CD005121.

Mita A, Ricordi C, Messinger S, Miki A, Misawa R, Barker S, Molano RD, Haertter R, Khan A, Miyagawa S, et al. 2010 Anti-proinflammatory effects of iodixanol (OptiPrep)based density gradient purification on human islet preparations. Cell Transplant 19: 1537-1546.

Mittal VK, Toledo-Pereyra LH, Sharma M, Ramaswamy K, Puri VK, Cortez JA, Gordon D. 1981. Acute portal hypertension and disseminated intravascular coagulation following pancreatic islet autotransplantation after subtotal pancreatectomy. Transplantation 31: 302-304.

Morel P, Kaufman DB, Field MJ, Lloveras JK, Matas AJ, Sutherland DE. 1992. Detrimental effect of prednisone on canine islet autograft function. Transplant Proc 24: 1048-1050.

Nano R, Clissi B, Melzi R, Calori G, Maffi P, Antonioli B, Marzorati S, Aldrighetti L, Freschi M, Grochowiecki T, et al. 2005. Islet isolation for allotransplantation: Variables associated with successful islet yield and graft function. Diabetologia 48: 906-912.

Nepom GT. 2005. Tetramer analysis of human autoreactive CD4-positive T cells. Adv Immunol 88: 51-71.

Nilsson B, Ekdahl KN, Korsgren O. 2011. Control of instant blood-mediated inflammatory reaction to improve islets of Langerhans engraftment. Curr Opin Organ Transplant 16: $620-626$.

Noguchi H, Ikemoto T, Naziruddin B, Jackson A, Shimoda M, Fujita Y, Chujo D, Takita M, Kobayashi N, Onaca N, et al. 2009. Iodixanol-controlled density gradient during islet purification improves recovery rate in human islet isolation. Transplantation 87: 1629-1635.

O'Gorman D, Kin T, Murdoch T, Richer B, McGhee-Wilson D, Ryan EA, Shapiro JA, Lakey JR. 2005. The standardization of pancreatic donors for islet isolations. Transplantation 80: 801-806.
O'Gorman D, Kin T, Imes S, Pawlick R, Senior P, Shapiro AM. 2010. Comparison of human islet isolation outcomes using a new mammalian tissue-free enzyme versus collagenase NB-1. Transplantation 90: 255-259.

Omer A, Duvivier-Kali VF, Trivedi N, Wilmot K, BonnerWeir S, Weir GC. 2003. Survival and maturation of microencapsulated porcine neonatal pancreatic cell clusters transplanted into immunocompetent diabetic mice. $\mathrm{Di}$ abetes 52: 69-75.

Owen RJ, Ryan EA, O'Kelly K, Lakey JR, McCarthy MC, Paty BW, Bigam DL, Kneteman NM, Korbutt GS, Rajotte RV, et al. 2003. Percutaneous transhepatic pancreatic islet cell transplantation in type 1 diabetes mellitus: Radiologic aspects. Radiology 229: 165-170.

Pedersen-Bjergaard U, Pramming S, Heller SR, Wallace TM, Rasmussen AK, Jorgensen HV, Matthews DR, Hougaard P, Thorsteinsson B. 2004. Severe hypoglycaemia in 1076 adult patients with type 1 diabetes: Influence of risk markers and selection. Diabetes Metab Res Rev 20: 479486.

Posselt AM, Bellin MD, Tavakol M, Szot GL, Frassetto LA, Masharani U, Kerlan RK, Fong L, Vincenti FG, Hering BJ, et al. 2010a. Islet transplantation in type 1 diabetics using an immunosuppressive protocol based on the anti-LFA-1 antibody efalizumab. Am J Transplant 10: 1870-1880.

Posselt AM, Szot GL, Frassetto LA, Masharani U, Tavakol M, Amin R, McElroy J, Ramos MD, Kerlan RK, Fong L, et al. 2010b. Islet transplantation in type 1 diabetic patients using calcineurin inhibitor-free immunosuppressive protocols based on T-cell adhesion or costimulation blockade. Transplantation 90: 1595-1601.

Pugliese A, Reijonen HK, Nepom J, Burke GW III. 2011. Recurrence of autoimmunity in pancreas transplant patients: Research update. Diabetes Manag (Lond) 1: 229238.

Rafael E, Tibell A, Ryden M, Lundgren T, Savendahl L, Borgstrom B, Arnelo U, Isaksson B, Nilsson B, Korsgren O, et al. 2008. Intramuscular autotransplantation of pancreatic islets in a 7-year-old child: A 2-year follow-up. Am J Transplant 8: 458-462.

Ricordi C, Lacy PE, Scharp DW. 1989. Automated islet isolation from human pancreas. Diabetes 38: 140-142.

Rossini AA. 2004. Autoimmune diabetes and the circle of tolerance. Diabetes 53: 267-275.

Saidi RF, Bradley J, Greer D, Luskin R, O'Connor K, Delmonico F, Kennealey P, Pathan F, Schuetz C, Elias N, et al. 2010. Changing pattern of organ donation at a single center: Are potential brain dead donors being lost to donation after cardiac death? Am J Transplant 10: $2536-2540$.

Scharp DW, Lacy PE, Santiago JV, McCullough CS, Weide LG, Falqui L, Marchetti P, Gingerich RL, Jaffe AS, Cryer $\mathrm{PE}$, et al. 1990. Insulin independence after islet transplantation into type I diabetic patient. Diabetes 39: 515-518.

Scharp DW, Swanson CJ, Olack BJ, Latta PP, Hegre OD, Doherty EJ, Gentile FT, Flavin KS, Ansara MF, Lacy PE. 1994. Protection of encapsulated human islets implanted without immunosuppression in patients with type I or type II diabetes and in nondiabetic control subjects. $\mathrm{Di}$ abetes 43: $1167-1170$.

Shapiro AM, Hao E, Lakey JR, Finegood D, Rajotte RV, Kneteman NM. 1998. Diabetogenic synergism in canine 
M. McCall and A.M. James Shapiro

islet autografts from cyclosporine and steroids in combination. Transplant Proc 30: 527.

Shapiro AM, Lakey JR, Ryan EA, Korbutt GS, Toth E, Warnock GL, Kneteman NM, Rajotte RV. 2000. Islet transplantation in seven patients with type 1 diabetes mellitus using a glucocorticoid-free immunosuppressive regimen. N Engl J Med 343: 230-238.

Shapiro AM, Ricordi C, Hering B. 2003. Edmonton's islet success has indeed been replicated elsewhere. Lancet 362: 1242.

Shapiro AM, Ricordi C, Hering BJ, Auchincloss H, Lindblad R, Robertson RP, Secchi A, Brendel MD, Berney T, Brennan DC, et al. 2006. International trial of the Edmonton protocol for islet transplantation. $N$ Engl J Med 355: $1318-1330$.

Sharma V, Andersen D, Thompson M, Woda BA, Stoff JS, Hartigan C, Rastellini C, Phillips D, Mordes JP, Rossini AA. 2006. Autoimmunity after islet-cell allotransplantation. N Engl J Med 355: 1397-1399.

Suzuki K, Bonner-Weir S, Trivedi N, Yoon KH, HollisterLock J, Colton CK, Weir GC. 1998. Function and survival of macroencapsulated syngeneic islets transplanted into streptozocin-diabetic mice. Transplantation 66: 21-28.

Szot GL, Lee MR, Tavakol MM, Lang J, Dekovic F, Kerlan RK, Stock PG, Posselt AM. 2009. Successful clinical islet isolation using a GMP-manufactured collagenase and neutral protease. Transplantation 88: 753-756.

The Collaborative Islet Transplant Registry. 2009. Annual Report, www.citregistryorg/reports/reports.htm.

Thompson DM, Meloche M, Ao Z, Paty B, Keown P, Shapiro RJ, Ho S, Worsley D, Fung M, Meneilly G, et al. 2011. Reduced progression of diabetic microvascular complications with islet cell transplantation compared with intensive medical therapy. Transplantation 91: 373-378.

Toronto Congenital Cardiac Centre for Adults (TCCCA) Team. 2009. The Collaborative Islet Transplant Registry 2009 Annual Report, www.citregistryorg/reports/ reports.htm.

Toso C, Vallee JP, Morel P, Ris F, Demuylder-Mischler S, Lepetit-Coiffe M, Marangon N, Saudek F, James Shapiro AM, Bosco D, et al. 2008. Clinical magnetic resonance imaging of pancreatic islet grafts after iron nanoparticle labeling. Am J Transplant 8: 701-706.

Toso C, Isse K, Demetris AJ, Dinyari P, Koh A, Imes S, Kin T, Emamaullee J, Senior P, Shapiro AM. 2009. Histologic graft assessment after clinical islet transplantation. Transplantation 88: 1286-1293.

Toso C, McCall M, Emamaullee J, Merani S, Davis J, Edgar R, Pawlick R, Kin T, Knudsen LB, Shapiro AM. 2010.
Liraglutide, a long-acting human glucagon-like peptide 1 analogue, improves human islet survival in culture. Transpl Int 23: 259-265.

Vaithilingam V, Tuch BE. 2011. Islet transplantation and encapsulation: An update on recent developments. Rev Diabet Stud 8: 51-67.

Vendrame F, Pileggi A, Laughlin E, Allende G, Martin-Pagola A, Molano RD, Diamantopoulos S, Standifer N, Geubtner K, Falk BA, et al. 2010. Recurrence of type 1 diabetes after simultaneous pancreas-kidney transplantation, despite immunosuppression, is associated with autoantibodies and pathogenic autoreactive CD4 T-cells. Diabetes 59: 947-957.

Villiger P, Ryan EA, Owen R, O’Kelly K, Oberholzer J, Al Saif F, Kin T, Wang H, Larsen I, Blitz SL, et al. 2005. Prevention of bleeding after islet transplantation: Lessons learned from a multivariate analysis of 132 cases at a single institution. Am J Transplant 5: 2992-2998.

Vincenti F, Larsen C, Durrbach A, Wekerle T, Nashan B, Blancho G, Lang P, Grinyo J, Halloran PF, Solez K, et al. 2005. Costimulation blockade with belatacept in renal transplantation. N Engl J Med 353: 770-781.

Warnock GL, Thompson DM, Meloche RM, Shapiro RJ, Ao Z, Keown P, Johnson JD, Verchere CB, Partovi N, Begg IS, et al. 2008. A multi-year analysis of islet transplantation compared with intensive medical therapy on progression of complications in type 1 diabetes. Transplantation 86: 1762-1766.

Williams PW. 1894. Notes on diabetes treated with extract and by grafts of sheep's pancreas. Br Med J: 1303-1304.

Witkowski P, Liu Z, Cernea S, Guo Q, Poumian-Ruiz E, Herold K, Hardy MA. 2006. Validation of the scoring system for standardization of the pancreatic donor for islet isolation as used in a new islet isolation center. Transplant Proc 38: 3039-3040.

Wszola M, Berman A, Fabisiak M, Domagala P, Zmudzka M, Kieszek R, Perkowska-Ptasinska A, Sabat M, Pawelec K, Kownacki L, et al. 2009. TransEndoscopic Gastric SubMucosa Islet Transplantation (eGSM-ITx) in pigs with streptozotocine induced diabetes-Technical aspects of the procedure-Preliminary report. Ann Transplant 14: $45-50$.

Yang Z, Chen M, Fialkow LB, Ellett JD, Wu R, Nadler JL. 2002. Survival of pancreatic islet xenografts in NOD mice with the theracyte device. Transplant Proc 34: 3349-3350.

Zeng Y, Torre MA, Karrison T, Thistlethwaite JR. 1994. The correlation between donor characteristics and the success of human islet isolation. Transplantation 57: 954-958. 


\section{$\&_{\mathrm{CSH}}^{\infty} \&$ Cold Spring Harbor

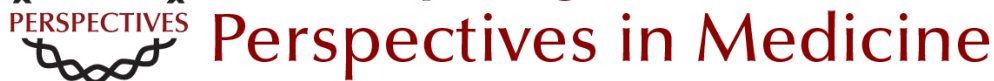

\section{Update on Islet Transplantation}

Michael McCall and A.M. James Shapiro

Cold Spring Harb Perspect Med 2012; doi: 10.1101/cshperspect.a007823 originally published online May 8, 2012

\section{Subject Collection Type I Diabetes}

The Pathogenesis and Natural History of Type 1 Diabetes

Mark A. Atkinson

\section{Do MHCII-Presented Neoantigens Drive Type 1}

Diabetes and Other Autoimmune Diseases? Philippa Marrack and John W. Kappler

Clinical Immunologic Interventions for the Treatment of Type 1 Diabetes Lucienne Chatenoud, Katharina Warncke and Anette-G. Ziegler

Update on Islet Transplantation Michael McCall and A.M. James Shapiro

Immunologic and Metabolic Biomarkers of $\beta$-Cell Destruction in the Diagnosis of Type 1 Diabetes Jasmin Lebastchi and Kevan C. Herold

Advancing Animal Models of Human Type 1 Diabetes by Engraftment of Functional Human Tissues in Immunodeficient Mice Michael A. Brehm, Alvin C. Powers, Leonard D. Shultz, et al.

Breakdown in Peripheral Tolerance in Type 1 Diabetes in Mice and Humans Lukas T. Jeker, Hélène Bour-Jordan and Jeffrey $A$. Bluestone

Antigen-Specific Therapeutic Approaches in Type 1 Diabetes

Xavier Clemente-Casares, Sue Tsai, Carol Huang, et al.
Humoral Autoimmunity in Type 1 Diabetes:

Prediction, Significance, and Detection of Distinct

Disease Subtypes

Massimo Pietropaolo, Roberto Towns and George S. Eisenbarth

Endoplasmic Reticulum Stress, Pancreatic $\beta$-Cell

Degeneration, and Diabetes Feroz R. Papa

Islet Autoantigens: Structure, Function, Localization, and Regulation Peter Arvan, Massimo Pietropaolo, David Ostrov, et al.

Environmental Triggers of Type 1 Diabetes Mikael Knip and Olli Simell

Generating $\beta$ Cells from Stem Cells--The Story So Far Matthias Hebrok

Antigen Targets of Type 1 Diabetes Autoimmunity Bart O. Roep and Mark Peakman

Connecting Type 1 and Type 2 Diabetes through Innate Immunity Justin I. Odegaard and Ajay Chawla

The Hygiene Hypothesis: An Explanation for the Increased Frequency of Insulin-Dependent Diabetes Jean-François Bach and Lucienne Chatenoud

For additional articles in this collection, see http://perspectivesinmedicine.cshlp.org/cgi/collection/ 